\title{
LATERAL EPICONDYLITIS OF THE ELBOW
}

Marcio Cohen ${ }^{1}$, Geraldo da Rocha Motta Filho²

\section{ABSTRACT}

Lateral epicondylitis, also known as tennis elbow, is a common condition that is estimated to affect $1 \%$ to $3 \%$ of the population. The word epicondylitis suggests inflammation, although histological analysis on the tissue fails to show any inflammatory process. The structure most commonly affected is the origin of the tendon of the extensor carpi radialis brevis and the mechanism of injury is associated with overloading. Nonsurgical treatment is the preferred method, and this in- cludes rest, physiotherapy, cortisone infiltration, platelet-rich plasma injections and use of specific immobilization. Surgical treatment is recommended when functional disability and pain persist. Both the open and the arthroscopic surgical technique with resection of the degenerated tendon tissue present good results in the literature.

Keywords - Tennis Elbow/pathology; Tennis Elbow/therapy; Tennis Elbow/surgery

\section{INTRODUCTION}

Lateral epicondylitis is a frequent cause of elbow pain and affects 1 to $3 \%$ of the adult population every year. Although it was first reported in 1873, by Runge, the association with the term "tennis elbow" was first made in 1883 , by Major ${ }^{(1,2)}$.

Today, it is clear that lateral epicondylitis is a degenerative disorder that compromises the extensor tendons originating from the lateral epicondyle, extending infrequently to the joint. Although the terms epicondylitis and tendinitis are used to describe "tennis elbow", histopathological studies like those of Nirschl characterize this condition not as an inflammatory condition but, rather, as a form of tendinosis with a fibroblastic and vascular response called angiofibroblastic degeneration of epicondylitis ${ }^{(3)}$.

Despite the classical description relating to practicing the sport of tennis, only 5 to $10 \%$ of the patients who present epicondylitis practice this sport $^{(4)}$. Thus, tendinosis of the elbow is more common among non-sports players. It occurs mostly in the fourth and fifth decades of life, affects both sexes similarly and is more frequent in the dominant arm.
As well as in tennis players, it may occur in people practicing other sports and has also been correlated with a variety of manual labor activities ${ }^{(3)}$. Lateral epicondylitis occurs initially through microlesions at the origin of the extensor musculature of the forearm, and most frequently affects the short radial extensor tendon of the carpus (SREC), which is located below the long radial extensor of the carpus (LREC) (Figure 1). According to Nirschl ${ }^{(5)}$, in $35 \%$ of the patients treated surgically in their series, not only was the SREC affected, but also $10 \%$ of the anterior face of the extensor aponeurosis.

\section{PATHOLOGY}

In the past, it was believed that epicondylitis was an inflammatory process. Perioperative inspection in most cases reveals homogenous grayish tissue with edema. This abnormality occurs in cases of tendinosis, irrespective of whether they are lateral, medial or posterior. Nirschl and Pettrone ${ }^{(3)}$, and also Regan et $a l^{(6)}$, made assessments under a microscope and found ruptures of the normal architecture of collagen fibers, with growth of fibroblasts and granulation tissue. These authors demonstrated that

1 - Physician at the Shoulder and Elbow Surgery Center, National Institute of Traumatology and Orthopedics (INTO), Rio de Janeiro, RJ, Brazil.

2 - Director-General of the National Institute of Traumatology and Orthopedics (INTO), Rio de Janeiro, RJ, Brazil.

Work performed at the National Institute of Traumatology and Orthopedics (INTO), Rio de Janeiro, RJ, Brazil.

Correspondence: Av. General San Martin, 300/702, 22441-010 Rio de Janeiro, RJ. E-mail: mcohen5@hotmail.com

Work received for publication: October 5, 2011; accepted for publication: December 1, 2011.

The authors declare that there was no conflict of interest in conducting this work 


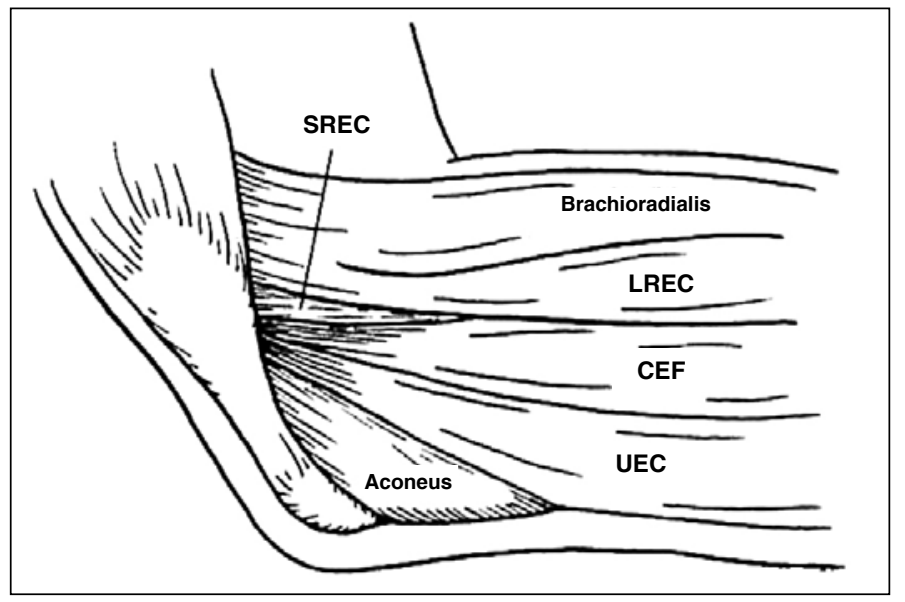

Figure 1 - Drawing of the anatomy of the lateral face of the elbow. The SREC is located below the common extensor of the fingers and the LREC.

these micro-ruptures were accompanied by partial healing and angiofibroblastic hyperplasia. The granulation tissue that forms is grayish and friable. Nonetheless, it needs to be emphasized that in the initial phase, epicondylitis may present inflammatory signs ${ }^{(3,6,7)}$. Nirschl ${ }^{(8)}$ previously classified lesions secondary to tendinous microtrauma in cases of lateral epicondylitis, into four stages. The first stage is inflammatory, reversible and without pathological alterations. The second stage is characterized by angiofibroblastic degeneration. The third stage is characterized by tendinosis associated with structural alteration (tendon tearing). In the fourth stage, in addition to the latter alterations, fibrosis and calcification are present.

\section{DIAGNOSIS}

The diagnosis is basically made by observing the patient's history and clinical examination. The main complaint consists of pain in the region of the lateral epicondyle extending to the dorsum of the forearm, along with incapacity to practice sports or do manual labor activities and activities of daily living. In general, the pain arises through activities that involve active extension or passive flexion of the wrist with the elbow extended.

\section{PHYSICAL EXAMINATION}

Palpation starts with identification of the lateral and medial epicondyles and the tip of the olecranon. On the lateral face, the origin of the extensor musculature of the wrist and fingers, the lateral ligament complex and the head of the radius are palpated. Pain located in the lateral epicondyle and at the origin of the extensor musculature of the wrist is suggestive of lateral epicondylitis or radial tunnel syndrome. The examination should continue with palpation of the head of the radius, in a depression just below the extensor musculature of the wrist. This is done during pronosupination, at varying degrees of flexion-extension, to assess its outline and integrity. The specific clinical test for lateral epicondylitis has the aim of reproducing the pain experienced by the patient. The test known as Cozen's test is done with the elbow flexed at $90^{\circ}$ and with the forearm in pronation. The patient is asked to perform active extension of the wrist against the resistance imposed by the examiner. The test result will be positive when the patients reports pain in the lateral epicondyle and at the origin of the extensor musculature of the wrist and fingers ${ }^{(9)}$.

The alternative test, known as Mill's test, is performed with the patient's hand closed, the wrist in dorsiflexion and the elbow extended. The examiner then forces the wrist into flexion and the patient is instructed to resist this movement. The test is positive if the patient feels pain in the lateral epicondyle ${ }^{(9)}$.

\section{COMPLEMENTARY EXAMINATIONS}

Anteroposterior, lateral and oblique radiographic evaluations show normal results in most cases, and are mainly useful for ruling out other abnormalities such as arthrosis, osteochondritis dissecans and intra-articular free bodies. Calcifications in the region of the lateral epicondyle are only infrequently present, occurring in approximately $22 \%$ of the cases, which according to some authors suggests a process that is refractory to closed treatment (Figure 2$)^{(8,10)}$.

Pomerance ${ }^{(11)}$ evaluated radiographs on the elbows of 271 patients with lateral epicondylitis. Only $16 \%$ of the patients presented some type of radiographic alteration, among which the most common was the presence of lateral calcification in $7 \%$ of the cases. Only two patients presented abnormalities that justified changes in their treatment, due to a diagnosis of osteochondritis dissecans of the capitellum. This author's conclusion from reviewing these cases was that radiography was a non-essential examination at the time of patients' initial presentation of lateral epicondylitis. Ultrasonography on the elbow is a simple auxiliary examination for assessing soft tissues, which might present abnormalities in cases of epicondylitis. However, its value is debatable because it is 


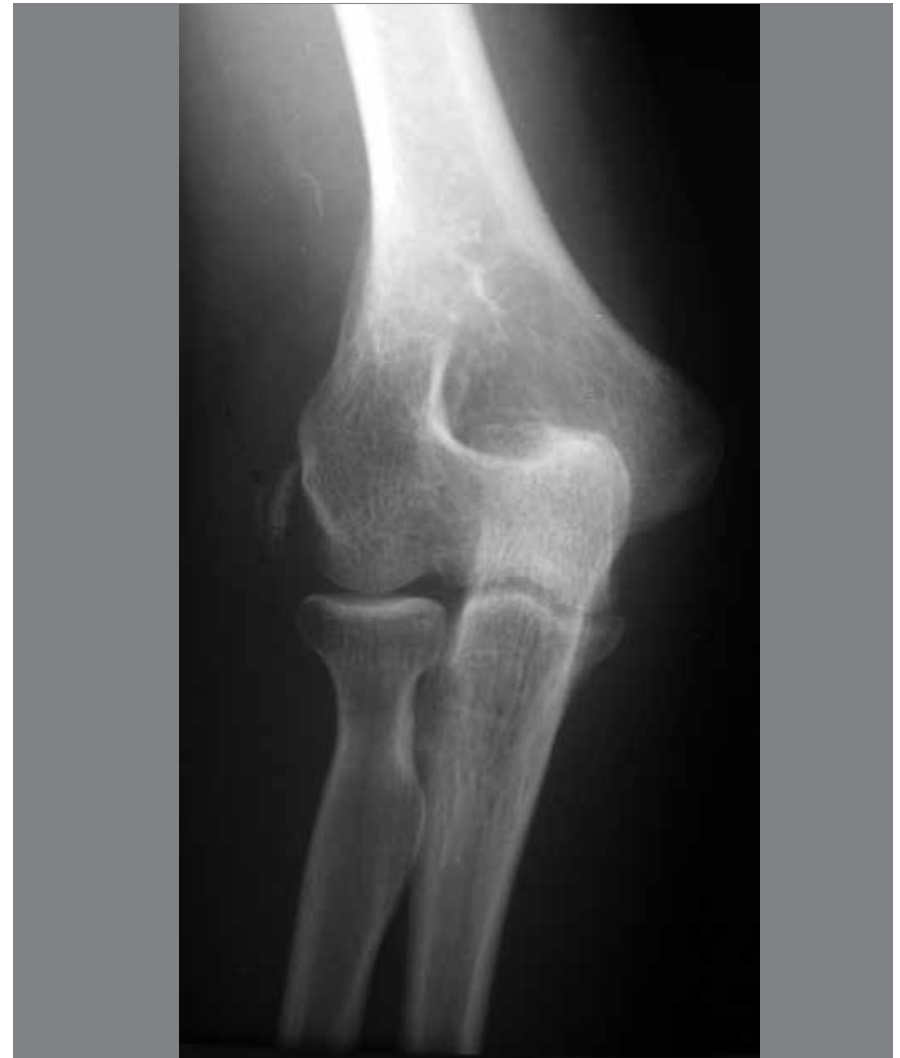

Figure 2 - Radiograph of the elbow, showing calcification of the lateral epicondyle.

examiner-dependent. Magnetic resonance imaging is an examination increasingly used in cases that are refractory to closed treatment of epicondylitis, since it assists in ruling out other pathological conditions and may also influence the surgical technique to be used for treating this tendinosis.

Potter et al ${ }^{(12)}$ evaluated cases of chronic lateral epicondylitis using magnetic resonance imaging and observed that there was an increase in the $\mathrm{T} 2$ signal at the origin of the SREC tendon in $50 \%$ of the patients. Aoki et $a l^{(13)}$ found an increase in the T2 signal at the origin of the SREC, at the lateral epicondyle, in six of their eleven patients with chronic lateral epicondylitis. Other abnormalities included a diffuse increase in the signal at the origin of the extensors, osteochondral fracture of the capitellum and presence of a ganglion at the radial nerve. These six patients were treated surgically using the technique of enucleation only at the location corresponding to the abnormality characterized from magnetic resonance imaging, i.e. at the origin of the SREC in the lateral cortical bone of the lateral epicondyle. All of these six patients achieved a clinical improvement. The authors' conclusion was that magnetic resonance imaging assisted in choosing the type of surgical treatment to be used.

\section{DIFFERENTIAL DIAGNOSIS}

There are conditions that may occur independently or in association with elbow tendinosis. Among the differential diagnoses, radial tunnel syndrome can be highlighted. This is characterized by compression of the posterior interosseous nerve and its diagnosis is essentially clinical, given that electromyography often produces normal results. Other differential diagnoses include cervicobrachialgia, rotator cuff injuries and joint abnormalities such as synovitis, intra-articular free bodies, post-traumatic osteoarthrosis and ligament injuries.

\section{CLOSED TREATMENT}

Patients presenting "tennis elbow" basically complain of pain. Therefore, pain control is the main objective of the initial treatment, through relative rest, which can be defined not as abstention from activity but, rather, as control over excesses. Use of plaster-cast immobilization is ineffective, given that the pain usually reappears when activities are resumed. Immobilization of the wrist also has little value, except in the reversible and inflammatory initial stage.

In relation to sports practice, the correct technique will enable better performance while preventing injuries. The sports correlated with lateral or medial epicondylitis include tennis, golf, sports using rackets in general, swimming and weight-lifting, among others.

Manual labor activities such as carpentry and other activities in which the hands are frequently used, such as typing, have also been correlated with epicondylitis.

Changing the sports or work activity is effective in controlling the pain. Use of non-steroidal anti-inflammatory drugs (NSAIDs), cryotherapy, ultrasound and laser are adjuvants for achieving analgesia. Since epicondylitis is a degenerative process, the benefits from using NSAIDs come from their analgesic effect and the synovitis that may be present initially. The efficiency of ultrasound has been assessed systematically, in comparison with placebo, without any statistical difference in the results ${ }^{(14)}$. Use of a functional immobilizer (brace) on the elbow has attracted a certain amount of popularity. Theoretically, because this limits the expansion of the extensor musculature in the proximal third of the forearm, it may diminish the force on vulnerable or sensitive areas. The brace generally has a width of five centimeters $(\mathrm{cm})$ and is placed 4 to 5 
$\mathrm{cm}$ distally to the epicondyle. Although there is some evidence that it is effective from a biomechanical point of view, there is not such evidence from a clinical point of view, as demonstrated by Kroslak and Murrell ${ }^{(15)}$.

Infiltration of corticosteroids may be indicated in cases in which, despite the physiotherapeutic treatment instituted, there is no improvement in the pain, thereby making it impossible for the patient to start doing rehabilitation exercises. The infiltration should be performed in the SREC, at a point just below and slightly distally to the lateral epicondyle. Performing more than two infiltrations may be harmful because of the adverse effects relating to peritendinous infiltration of corticosteroids, such as necrosis, tissue atrophy and consequent tendon tearing. To avoid these complications, the infiltration should not be intratendinous or very superficial (Figure 3$)^{(16)}$.

There are few randomized studies that could be used as parameters for making decisions regarding use of corticosteroids for treating lateral epicondylitis of the elbow. Nonetheless, the data that exists suggest that infiltration is superior to other forms of treatment, from short-term assessments of up to six months ${ }^{(17)}$. In the systematic evaluation conducted by Smidt et $a l^{(17)}$, there was no evidence of significant differences over the medium and long terms with regard to superiority of local injections of corticosteroid. Likewise, the data in the literature do not allow any conclusion to be reached regarding the ideal type and dosage of corticosteroid for use in infiltrations.

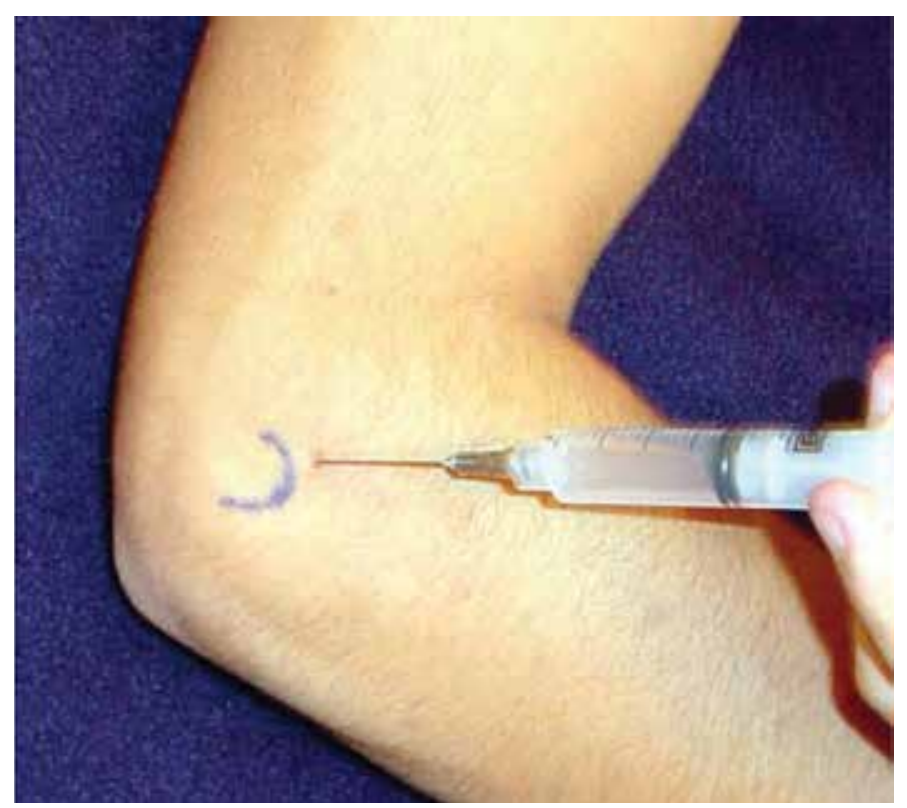

Figure 3 - Illustration of the ideal location for performing infiltration with corticosteroids for lateral epicondylitis.
Not long ago, infiltration with botulinum toxin was proposed as a new treatment method. Its principle consists of allowing tissue healing in an environment with lower tension, through partial paralysis of the extensors, caused by the anticholinergic action of this medication. Two recently published studies compared injection of botulinum toxin with placebo. Wong et al ${ }^{(18)}$ reported better results relating to pain after a 12-week period, in a group that received medication, compared with placebo. Hayton et al $l^{(19)}$ did not observe any differences after three months. In both of these studies, the weakness of finger and wrist extension caused by the botulinum toxin affected the manual workers in some manner.

Independent of the treatment instituted, once control over the pain has been achieved, patients can start to perform exercises aimed at stretching and gaining joint range of motion for the wrist and elbow, followed by isometric and isokinetic exercises. If there is no pain, the process of muscle reinforcement can be started, and use of a brace to control muscle expansion is recommended. Patients perform exercises and will be authorized to return to sports practice or manual labor activities when they are capable to performing repeated exercises until reaching tiredness, without occurrence of pain, and when they have attained muscle strength comparable with the levels that existed prior to the epicondylitis.

It needs to be emphasized again that there are no studies comparing stretching exercises and muscle strengthening with placebo use. In the case of returning to tennis practice, it is essential that patients should receive guidance. The circumference of the racket handle should be equal to the distance from the proximal palm crease to the tip of the ring finger along its radial edge (Figure 4). Measures capable of diminishing the trembling that is transmitted to the elbow, through using light rackets that are preferably made of graphite, with lower cording pressure or greater numbers of fibers.

Another form of treatment is shockwaves, and the efficacy of such treatment has been studied. Pettrone and $\mathrm{McCall}^{(20)}$ observed a reduction of at least $50 \%$ in the degree of pain, in $64 \%$ of their patients who underwent this type of therapy. On the other hand, Haake et $a l^{(21)}$ demonstrated in a prospective study that shockwaves were not effective. In a review of the literature, Buchbinder et al ${ }^{(22)}$ concluded that the benefit from shockwave therapy for lateral epicondylitis was minimal. Recently, great emphasis has been 


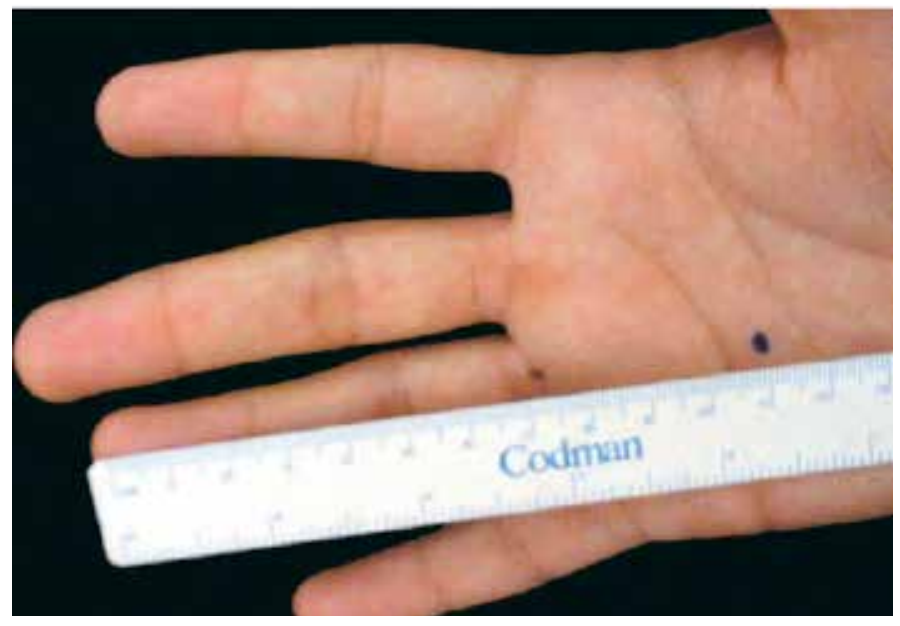

Figure 4 - Parameters for determining the ideal diameter for a tennis racket handle.

placed on infiltration with platelet-rich plasma (PRP) as another alternative form of closed treatment. Starting from the principle that the histopathological findings from lateral epicondylitis are related to tendon degeneration, the ideal treatment would be based on biological stimulation of tendon repair. PRP is an autologous product created from centrifugation of the patient's own blood, which contains large concentrations of growth factors derived from platelets. It is believed that local injection of PRP may diminish the pain relating to this pathological condition, through an inflammatory reaction with consequent angiogenesis, fibroplasia, collagen synthesis and tissue remodeling ${ }^{(23)}$. However, there is great controversy regarding the use of PRP in orthopedic practice, and few statistically significant studies exist. On the other hand, Gosens et al ${ }^{(24)}$ recently published a level-of-evidence study comparing local infiltration to treat lateral epicondylitis using PRP and corticosteroids, with a two-year follow-up. A group of 100 patients was randomized to receive an injection of either PRP or corticosteroid, and the conclusion was that the group treated with local injection of PRP achieved greater pain relief and functional improvement than seen in the other group.

\section{SURGICAL TREATMENT}

Patients who undergo correct rehabilitation for a period of not less than nine months but without the pain being brought under control are candidates for surgery, especially if the closed treatment performed has included three or more unsuccessful infiltrations and when the process is a factor limiting the patient's activities of daily living.
Among the surgical techniques that exist are the open, percutaneous and arthroscopic procedures. Although there are several studies in the literature with results from these techniques, there are few that have compared the techniques with each other.

The open surgical technique that is most used is the one described and made popular by Nirschl. This consists of identifying and resecting the area of tendinosis, which may include all of the origin of the SREC and, in some cases, the anteromedial aponeurosis of the common extensor of the fingers (CEF) (Figure 5) ${ }^{(25,26)}$. Once the diseases tissue has been removed, there will be a defect of variable size. It is useful to promote stimulation of blood circulation at this site by means of making two or three bone orifices in the lateral epicondyle, thus favoring formation of a hematoma at this location. Suturing the remainder of the SREC to the aponeurosis of the common extensor is unnecessary and, if performed, this tends to block complete extension of the elbow. On the other hand, suturing the posterolateral edge of the LREC to the aponeurosis of the common extensor is recommended.

The technique originally described by $\mathrm{Nirschl}^{(3)}$ in 1979 has been modified over the course of time. Today, smaller incisions are made (between 1.5 and $3 \mathrm{~cm}$ ) and only one bone perforation in the anterolateral region of the lateral condyle, rather than strictly in the lateral epicondyle $^{(27)}$. The elbow is initially immobilized for around seven days. Isotonic and isokinetic exercises are started after three weeks by using the functional immobilizer to control muscle expansion: this should be kept in use for two to three months, even during activities of daily living. The return to sports practice should be gradual, beginning after eight weeks and attaining levels close to ideal after around six months. Dunn et $a l^{(27)}$ observed that $84 \%$ of their results were excellent or good, among 92 cases treated with a modified version of the original technique, which they described as mini-open. The most important point from their study was the minimum follow-up of 10 years, thus showing good results over the long term.

Like the open technique, arthroscopic surgery also has the aim of identifying and resecting tendinosis (Figure 6). Some authors have argued that this technique is advantageous, since it allows viewing and treatment of associated intra-articular pathological conditions, despite increasing the duration of surgery, the cost and the risk of neurovascular lesions. Studies on 


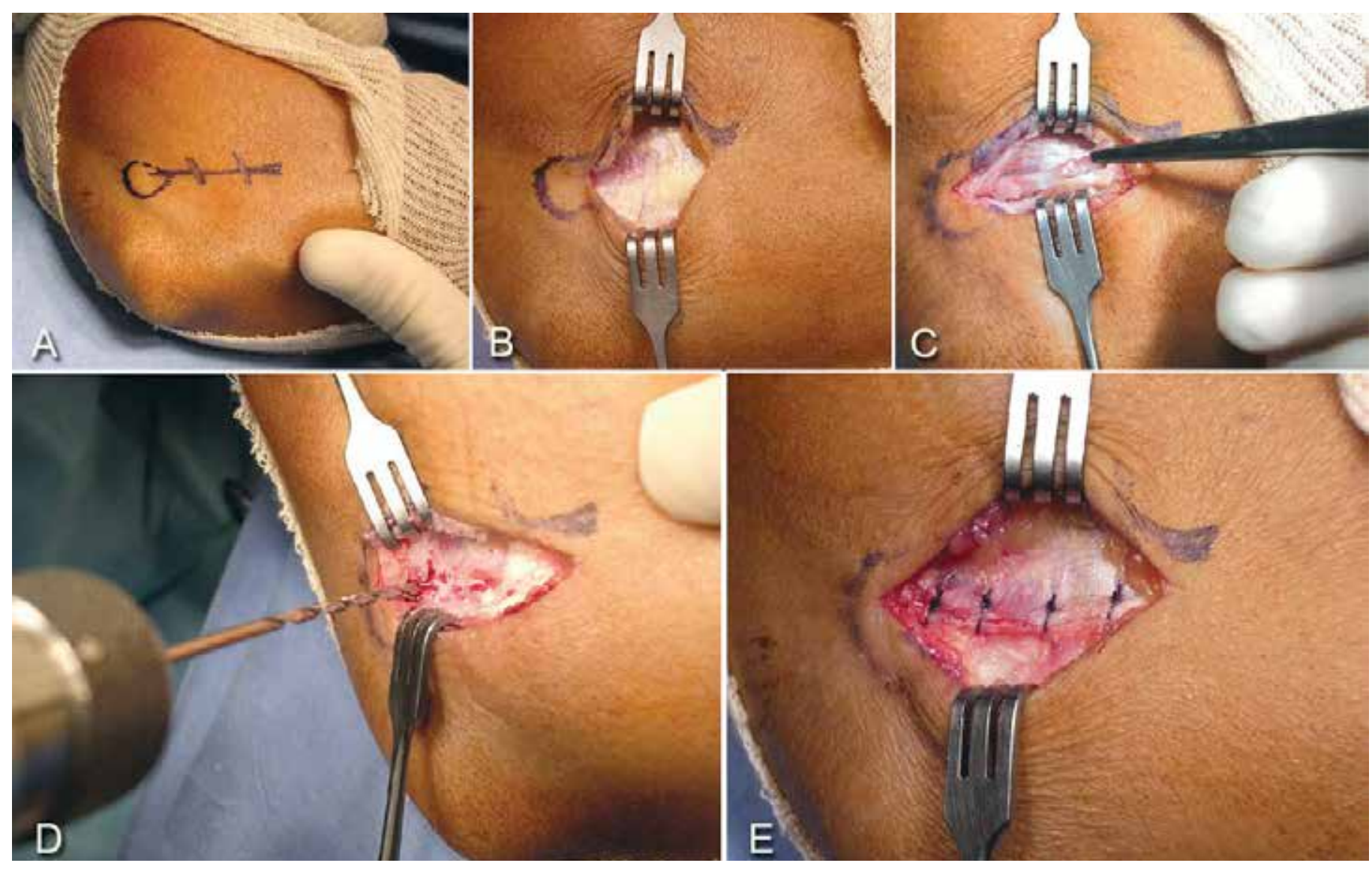

Figure 5 - Surgical technique used for treating lateral epicondylitis: (A) surgical access; (B) view of the gap between the SREC and the extensor aponeurosis; (C) view of tendinosis of the SREC after opening the extensor aponeurosis; (D) construction of a bone orifice in the lateral epicondyle to promote local blood circulation; (E) suturing of the gap between the SREC and the extensor aponeurosis.

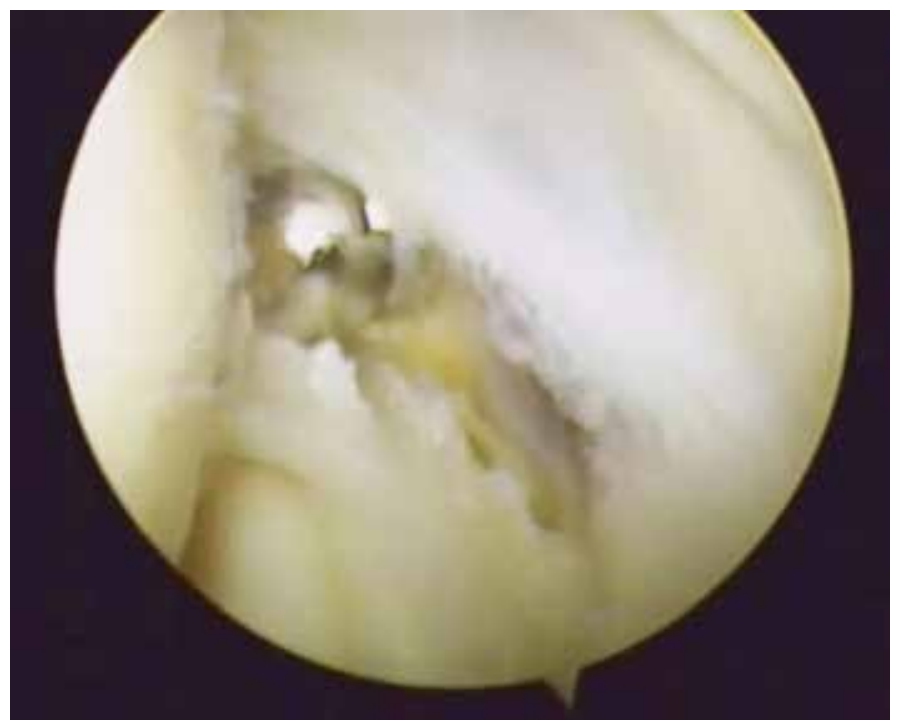

Figure 6 - Arthroscopic image of the elbow showing debridement of the SREC tendon after removal of the capsule.

cadavers have demonstrated the efficacy of resection at the origin of the SREC and CEF using the arthroscopic technique, without creating iatrogenic posterolateral instability ${ }^{(28)}$. Baker and Baker ${ }^{(29)}$ presented a high rate of satisfaction from arthroscopic treatment on $30 \mathrm{pa}-$ tients who were reassessed after a minimum follow-up of 106 months. Peart et al ${ }^{(30)}$ compared the open technique with arthroscopy, although this was by means of a retrospective study and not a randomized study. They did not find any statistically significant differences, although in the group treated using the arthroscopic technique, the time taken to return to work activities and the time taken for physiotherapy were shorter.

\section{COMPLICATIONS}

Complications relating to closed treatment are rare. In surgery, the lateral collateral ligament needs to be protected given the iatrogenic posterolateral instability of the elbow.

\section{FINAL REMARKS}

Despite the name, humeral epicondylitis is a non-inflammatory form of tendinopathy. Lateral epicondylitis originates in the extensors. The etiology is related to tendon overload, and this condition is dealt with prominently in the literature. The diagnosis is 
eminently clinical, and complementary examinations are needed essentially to conduct investigative studies and to rule out other diagnoses. Closed treatment is preferred, given that most patients improve through this. Infiltration with PRP seems to be a further alternative for treating lateral epicondylitis, although there is a need for additional controlled clinical studies.

In patients in whom the symptoms persist for a long time despite closed treatment, surgical treatment should be considered. This presents high rates of excellent and good results. We find it strange that such a small number of scientific studies respecting the currently recommended scientific criteria exist in relation to such a frequent disorder. For this reason, we are unable to establish specific protocols for treating lateral epicondylitis.

\section{REFERENCES}

1. Runge F. Zur Gênese and behandlung des schreibekrampfes. Berliner Klin Wchnschr. 1873;10:245-8.

2. Major HP. Lawn-tennis elbow. BMJ. 1883;2:557.

3. Nirschl RP, Pettrone FA. Tennis elbow. The surgical treatment of lateral epicondylitis. J Bone Joint Surg Am. 1979;61(6):832-9.

4. Boyer MI, Hastings $\mathrm{H}$ 2nd. Lateral tennis elbow: "Is there any science out there?". J Shoulder Elbow Surg. 1999;8(5):481-91.

5. Nirschl RP. Muscle and tendon trauma: tennis elbow tendinosis. In: Morrey BF. The elbow. Philadelphia: Saunders; 2000. p. 523-35.

6. Regan W, Wold LE, Coonrad R, Morrey BF. Microscopic histopathology of chronic refractory lateral epicondylitis. Am J Sports Med. 1992;20(6):746-9.

7. Kraushaar BS, Nirschl RP. Tendinosis of the elbow (tennis elbow). Clinical features and findings of histological, immunohistochemical, and electron microscopy studies. J Bone Joint Surg Am. 1999;81(2):259-78.

8. Nirschl RP. Elbow tendinosis/tennis elbow. Clin Sports Med. 1992;11(4):851-70.

9. Motta Filho GR. Cotovelo. In Barros Filho TEP, Lech O, editores. Exame físico em ortopedia, São Paulo, Sarvier; 2001. p.138-56.

10. Jobe FW, Ciccotti MG. Lateral and medial epicondylitis of the elbow. J Am Acad Orthop Surg. 1994;2(1):1-8.

11. Pomerance J. Radiographic analysis of lateral epicondylitis. J Shoulder Elbow Surg. 2002;11(2):156-7.

12. Potter HG, Hannafin JA, Morwessel RM, DiCarlo EF, O'Brien SJ, Altchek DW. Lateral epicondylitis: correlation of MR imaging, surgical, and histopathologic findings. Radiology. 1995;196(1):43-6.

13. Aoki M, Wada T, Isogai S, Kanaya K, Aiki H, Yamashita T. Magnetic resonance imaging findings of refractory tennis elbows and their relationship to surgical treatment. J Shoulder Elbow Surg. 2005;14(2):172-7.

14. D'Vaz AP, Ostor AJ, Speed CA, Jenner JR, Bradley M, Prevost AT,et al. Pulsed low-intensity ultrasound therapy for chronic lateral epicondylitis: a randomized controlled trial. Rheumatology (Oxford). 2006;45(5):566-70.

15. Kroslak M, Murrell GAC. Tennis elbow counterforce bracing. Techn Shoulder Elbow Surg. 2007;8:75-9.

16. Cole BJ, Schumacher HR Jr. Injectable corticosteroids in modern practice. J Am Acad Orthop Surg. 2005;13(1):37-46.

17. Smidt N, Assendelft WJ, van der Windt DA, Hay EM, Buchbinder R, Bouter LM. Corticosteroid injections for lateral epicondylitis: a systematic review. Pain. 2002;96(1-2):23-40.
18. Wong SM, Hui AC, Tong PY, Poon DW, Yu E, Wong LK. Treatment of lateral epicondylitis with botulinum toxin: a randomized, double-blind, placebo-controlled trial. Ann Intern Med. 2005;143(11):793-7.

19. Hayton MJ, Santini AJ, Hughes PJ, Frostick SP, Trail IA, Stanley JK. Botulinum toxin injection in the treatment of tennis elbow. A double-blind, randomized, controlled, pilot study. J Bone Joint Surg Am. 2005;87(3):503-7.

20. Pettrone FA, McCall BR. Extracorporeal shock wave therapy without local anesthesia for chronic lateral epicondylitis. J Bone Joint Surg Am. 2005;87(6):1297-304.

21. Haake M, König IR, Decker T, Riedel C, Buch M, Müller HH. Extracorporeal shock wave therapy in the treatment of lateral epicondylitis: a randomized multicenter trial. J Bone Joint Surg Am. 2002;84(11):1982-91.

22. Buchbinder R, Green SE, Youd JM, Assendelft WJ, Barnsley L, Smidt N. Shock wave therapy for lateral elbow pain. Cochrane Database Syst Rev. 2005; (4):CD003524.

23. Foster TE, Puskas BL, Mandelbaum BR, Gerhardt MB, Rodeo SA. Platelet-rich plasma: from basic science to clinical applications. Am J Sports Med. 2009;37(11):2259-72.

24. Gosens T, Peerbooms JC, van Laar W, den Oudsten BL. Ongoing positive effect of platelet-rich plasma versus corticosteroid injection in lateral epicondylitis: a double-blind randomized controlled trial with 2-year follow-up. Am J Sports Med. 2011;39(6):1200-8.

25. Coonrad RW, Hooper WR. Tennis elbow: its course, natural history, conservative and surgical management. J Bone Joint Surg Am. 1973;55(6):1177-82.

26. Nirschl RP. Lateral and medial epicondylitis. In Morrey BF, editor. Master techniques in orthopedic surgery: the elbow. Philadelphia: Lippincott Williams \& Wilkins; 1994. p. 129-48.

27. Dunn JH, Kim JJ, Davis L, Nirschl RP. Ten- to 14-year follow-up of the Nirschl surgical technique for lateral epicondylitis. Am J Sports Med. 2008;36(2):261-6.

28. Kuklo TR, Taylor KF, Murphy KP, Islinger RB, Heekin RD, Baker CL Jr. Arthroscopic release for lateral epicondylitis: a cadaveric model. Arthroscopy. 1999;15(3):259-64.

29. Baker CL Jr, Baker CL 3rd. Long-term follow-up of arthroscopic treatment of lateral epicondylitis. Am J Sports Med. 2008;36(2):254-60.

30. Peart RE, Strickler SS, Schweitzer KM Jr. Lateral epicondylitis: a comparative study of open and arthroscopic lateral release. Am J Orthop (Belle Mead NJ). 2004;33(11):565-7. 